\title{
Reidemeister number of any automorphism of a Gromov hyperbolic group is infinite
}

\author{
Alexander Fel'shtyn*
}

\begin{abstract}
We show that the number of twisted conjugacy classes is infinite for any automorphism of non-elementary, Gromov hyperbolic group [9]. An analog of Selberg theory for twisted conjugacy classes is proposed.
\end{abstract}

\section{Introduction}

Let $G$ be a finitely generated group and $\phi: G \rightarrow G$ an endomorphism. Two elements $\alpha, \alpha^{\prime} \in G$ are said to be $\phi-$ conjugate iff there exists $\gamma \in G$ with

$$
\alpha^{\prime}=\gamma \alpha \phi(\gamma)^{-1}
$$

We shall write $\{x\}_{\phi}$ for the $\phi$-conjugacy class of the element $x \in G$. The number of $\phi$-conjugacy classes is called the Reidemeister number of an endomorphism $\phi$, denoted by $R(\phi)$. If $\phi$ is the identity map then the $\phi$-conjugacy classes are the usual conjugacy classes in the group $G$.

We note that $R(\phi)$ is infinite if group $G$ is free Abelian and the action of $\phi$ on $G$ has 1 as eigenvalue [6].

In [5] we have conjectured that the Reidemeister number is infinite as long as the endomorphism $\phi$ is injective and the group $G$ has exponential growth.

In this paper we prove our conjecture for any automorphism of any non-elementary (i.e. not virtually cyclic), Gromov hyperbolic group. We also prove some generalisations of this result.

Main result of this paper has topological counterpart. Let $X$ to be a connected, compact polyhedron and $f: X \rightarrow X$ to be a continuous map. Let $p: \tilde{X} \rightarrow X$ be the universal cover of $X$ and $\tilde{f}: \tilde{X} \rightarrow \tilde{X}$ a lifting of $f$, i.e. $p \circ \tilde{f}=f \circ p$. Two liftings $\tilde{f}$ and $\tilde{f}^{\prime}$ are called conjugate if there is a element $\gamma$ in the deck transformation group $\Gamma \cong \pi_{1}(X)$ such that $\tilde{f}^{\prime}=\gamma \circ \tilde{f} \circ \gamma^{-1}$. The subset $p(F i x(\tilde{f})) \subset F i x(f)$ is called the fixed point class of $f$ determined by the lifting class $[\tilde{f}]$. Two fixed points $x_{0}$ and $x_{1}$ of $f$ belong to the same fixed point class iff there is a path $c$ from $x_{0}$ to $x_{1}$

*Part of this work was conducted during author stay in Universite Paul Sabatier, Toulouse and Ohio State University, Columbus 
such that $c \cong f \circ c$ (homotopy relative endpoints). This fact can be considered as an equivalent definition of a non-empty fixed point class. Every map $f$ has only finitely many non-empty fixed point classes, each a compact subset of $X$.

A fixed point class is called essential if its index is nonzero. The number of lifting classes of $f$ (and hence the number of fixed point classes, empty or not) is called the Reidemeister number of $f$, denoted $R(f)$. This is a positive integer or infinity. The number of essential fixed point classes is called the Nielsen number of $f$, denoted by $N(f)$. The Nielsen number is always finite.

Theorem 3 implies that the topological Reidemeister number $R(f)$ is infinite for a homeomorphism $f$ of a compact polyhedron $X$ with a non-elementary, Gromov hyperbolic fundamental group $\pi_{1}\left(X, x_{0}\right)$.

In the case of pseudo-Anosov homeomorphisms of surfaces we can develop an analog of the Selberg theory and we obtain an asymptotic expansion for the number of twisted conjugacy classes or for the number of Nielsen fixed point classes whose norm is at most $x$.

The author would like to thank M. Bridson, B. Bowditch, D. Burghelea, M. Davis, R. Grigorchyk, M. Gromov, G. Levitt, M. Lustig, Ch. Epstein, R. Hill, L. Potyagailo, for stimulating discussions and comments. The author would like to thank Laboratoire de Matematiques Emille Picard, Toulouse and the Departments of Mathematics, Ohio State University for their kind hospitality and support.

\section{Twisted conjugacy classes and Reidemeister num- ber of group endomorphism}

Lemma 1 If $G$ is a group and $\phi$ is an endomorphism of $G$ then an element $x \in G$ is always $\phi$-conjugate to its image $\phi(x)$.

PROOF. If $\gamma=x^{-1}$, then one has immediately $\gamma x=\phi(x) \phi(\gamma)$. The existence of a $\gamma$ satisfying this equation implies that $x$ and $\phi(x)$ are $\phi$-conjugate.

The mapping torus $M(\phi)$ of the group endomorphism $\phi: G \rightarrow G$ is obtained from group $G$ by adding a new generator $z$ and adding the relations $z^{-1} g z=\phi(g)$ for all $g \in G$. This means that $M(\phi)$ is a semi-direct product of $G$ with $Z$.

Lemma 2 Two elements $x, y$ of $G$ are $\phi$-conjugate if and only if $x z$ and $y z$ are conjugate in the usual sense in $M(\phi)$. Therefore $R(\phi)$ is the number of usual conjugacy classes in the coset $G \cdot z$ of $G$ in $M(\phi)$.

ProOF. If $x$ and $y$ are $\phi$-conjugate, then there is a $\gamma \in G$ such that $\gamma x=y \phi(\gamma)$. This implies $\gamma x=y z \gamma z^{-1}$ and therefore $\gamma(x z)=(y z) \gamma$. So $x z$ and $y z$ are conjugate in the usual sense in $M(\phi)$. Conversely suppose $x z$ and $y z$ are conjugate in $M(\phi)$. Then there is a $\gamma z^{n} \in M(\phi)$ with $\gamma z^{n} x z=y z \gamma z^{n}$. From the relation $z x z^{-1}=\phi(x)$, we obtain $\gamma \phi^{n}(x) z^{n+1}=y \phi(\gamma) z^{n+1}$ and therefore $\gamma \phi^{n}(x)=y \phi(\gamma)$. This shows that $\phi^{n}(x)$ and $y$ are $\phi$-conjugate. However, by lemma $1, x$ and $\phi^{n}(x)$ are $\phi$-conjugate, so $x$ and $y$ must be $\phi$-conjugate. 
Lemma 3 (T.Delzant) Let $J$ be a non-elementary, Gromov hyperbolic group. Let $K$ be a normal subgroup with abelian quotient. Then every coset $C$ of $J \bmod K$ contains infinitely many conjugacy classes.

Proof. (See [8]). Fix $u$ in the coset $C$ under consideration. Suppose for a moment that we can find $c, d \in K$, generating a free group of rank 2 , such that $u c^{\infty} \neq c^{-\infty}$ and $u d^{\infty} \neq d^{-\infty}$ (recall that we denote $g^{-\infty}=\lim _{n \rightarrow+\infty} g^{-n}$ for $g$ of infinite order). Consider $x_{k}=c^{k} u c^{k}$ and $y_{k}=d^{k} u d^{k}$. For $k$ large, the above inequalities imply that these two elements have infinite order, and do not generate a virtually cyclic group because $x_{k}^{+\infty}$ and $x_{k}{ }^{-\infty}$ (respectively $y_{k}{ }^{+\infty}$ and $\left.y_{k}{ }^{-\infty}\right)$ is close to $c^{+\infty}$ and $c^{-\infty}$ ( respectively $d^{+\infty}$ and $\left.d^{-\infty}\right)$. Fix $k$, and consider the elements $z_{n}=x_{k}{ }^{n+1} y_{k}{ }^{-n}$. They belong to the coset $C$, because $J / K$ is abelian, and their stable norm goes to infinity with $n$. Therefore $C$ contains infinitely many conjugacy classes. Let us now construct $c, d$ as above. Choose $a, b \in K$ generating a free group of rank 2 . We first explain how to get $c$. There is a problem only if $u a^{\infty}=a^{-\infty}$ and $u b^{\infty}=b^{-\infty}$. In that case there exists integers $p, q$ with $u a^{p} u^{-1}=a^{-p}$ and $u b^{q} u^{-1}=b^{-q}$. We take $c=a^{p} b^{q}$, noting that $u c u^{-1}=a^{-p} b^{-q}$ is different from $c^{-1}=b^{-q} a^{-p}$.

Once we have $c$, we choose $c^{*} \in K$ with $\left\langle c, c^{*}\right\rangle$ free of rank 2 , and we obtain $d$ by applying the preceding argument using $c^{*}$ and $c c^{*}$ instead of $a$ and $b$. The group $\langle c, d\rangle$ is free of rank 2 because $d$ is a positive word in $c^{*}$ and $c c^{*}$.

\subsection{Automorphisms of Gromov hyperbolic groups}

Let now $\phi$ be an automorphism of the Gromov hyperbolic group $G$ and let || $\mid$ denote the word metric with respect to some finite generating set for $G$. The automorphism $\phi$ is called hyperbolic if there is an integer $m$ and a number $\lambda>1$ such that, for all $g \in G$ we have $\max \left(\left\|\phi^{m}(g)\right\|,\left\|\phi^{-m}(g)\right\|\right) \geq \lambda\|g\|$. For example a pseudo-Anosov homeomorphism of a closed surface of genus larger then one induces a hyperbolic automorphism on the level of fundamental group. Also, an automorphism of finitely generated free group with no nontrivial periodic conjugacy classes is hyperbolic.

Lemma 4 [ The mapping torus $M(\phi)$ of a hyperbolic automorphism $\phi$ is Gromov hyperbolic group.

Theorem 1 The Reidemeister number $R(\phi)$ is infinite if group $G$ is Gromov hyperbolic, non-elementary, and $\phi$ is hyperbolic automorphism.

Proof The proof immediately folows from lemmas 2, 3 and 4 .

Let us consider an outer automorphism $\Phi \in$ Out $G$ corresponding to automorphism of $\phi \in A u t G$ and viewed as a collection of ordinary automorphisms $\alpha \in$ Aut $G$. We define $\alpha, \beta \in \Phi$ to be isogredient if $\beta=i_{h} \cdot \alpha \cdot i_{h}{ }^{-1}$ for some $h \in G$, with $i_{h}(g)=h g h^{-1}$.

Lemma 5 [8] The set $S(\Phi)$ of isogredience classes is infinite if group $G$ is Gromov hyperbolic, non-elementary, and $\Phi$ has finite order in the group OutG. 
Proof Let $J$ be the subgroup of $A u t G$ consisting of all automorphisms whose image in Out $G$ is a power of $\Phi$. The exact sequence $1 \rightarrow K \rightarrow J \rightarrow<\Phi>\rightarrow 1$, with $K=G /$ Center and $\langle\Phi\rangle$ finite, shows that $J$ is hyperbolic, non-elementary. The set of automorphisms $\alpha \in \Phi$ is a coset of $J \bmod K$. If $\alpha, \beta \in \Phi$ are isogredient they are conjugate in $J$. The proof is therefore concluded by applying lemma 3 .

Lemma 6 河] The set $S(\Phi)$ of isogredience classes is infinite if group $G$ is a free group $F_{n}$ and $\Phi \in O$ Out $\left(F_{n}\right)$ fixes a nontrivial conjugacy class.

Theorem 2 8] For any $\Phi \in$ Out $G$, with $G$ Gromov hyperbolic, non-elementary, the set $S(\Phi)$ of isogredience classes is infinite.

Proof We describe main steps of the proof. By lemma 5, we may assume that $\Phi$ has infinite order. By Paulin's theorem [11] $\Phi$ preserves some $R$-tree $T$ with nontrivial minimal small action of $G$ ( recall that an action of $G$ is small if all ars stabilisers are virtually cyclic; the action of $G$ on $T$ is always irreducible( no global fixed point, no invariant line, no invariant end)). This means that there is an $R$-tree $T$ equipped with an isometric action of $G$ whose length function satisfies $l \cdot \Phi=\lambda l$ for some $\lambda \geq 1$.

Step 1. Suppose $\lambda=1$. Then $S(\Phi)$ is infinite.

Step 2. Suppose $\lambda>1$. Assume that arc stabilisers are finite, and there exists $N_{0} \in N$ such that, for every $Q \in T$, the action of $S t a b Q$ on $\pi_{o}(T-Q)$ has at most $N_{0}$ orbits. Then $S(\Phi)$ is infinite.

Step 3. If $\lambda>1$, then $T$ has finite arc stabilisers. If $\lambda>1$ then from work of Bestwina-Feighn [2] it follows that there exists $N_{0} \in N$ such that, for every $Q \in T$, the action of $S t a b Q$ on $\pi_{o}(T-(Q))$ has at most $N_{0}$ orbits.

Theorem 3 The Reidemeister number $R(\phi)$ is infinite if group $G$ is Gromov hyperbolic, non-elementary, and $\phi$ is any automorphism of $G$.

Proof By definition, the automorphisms $\beta=i_{m} \cdot \alpha$ and $\gamma=i_{n} \cdot \alpha$ are isogredient if and only if there exists $g \in G$ with $\gamma=i_{g} \cdot \beta \cdot i_{g}{ }^{-1}$,or equivalently $n=g m \alpha\left(g^{-1}\right) c$ with $c$ in center of $G$. So, the set $S(\Phi)$ of isogredience classes of automorphisms representing $\Phi$ may be identified to the set of twisted conjugacy classes of $G \bmod$ its center. If $\phi$ is automorphism of finite order in $A u t G$, then the theorem immediately follows from lemma 5 . If an automorphism $\phi$ has infinite order in $A u t G$ then theorem follows from theorem 2 .

\section{$1.2 \quad$ The Co-Hopf property}

A group $G$ is called co-Hopf if every monomorphism of $G$ into itself is an isomorphism. It is fairly immediate to see that a freely decomposable group is not co-Hopf.

Lemma 7 (14) Let $G$ be a non-elementary, torsion-free, Gromov hyperbolic group. Then $G$ is co-Hopf if and only if $G$ is freely indecomposable. 
Theorem 4 The Reidemeister number $R(\phi)$ is infinite if group $G$ is Gromov hyperbolic, non-elementary,torsion free, freely indecomposable and $\phi$ is any monomorphism of $G$ into itself.

Proof The proof follows from lemma 7 and theorem 3 .

\subsection{Reduction to Injective Endomorphisms}

Let $G$ be a group and $\phi: G \rightarrow G$ an endomorphism. We shall call an element $x \in G$ nilpotent if there is an $n \in \mathbb{N}$ such that $\phi^{n}(x)=i d$. Let $N$ be the set of all nilpotent elements of $G$.

Lemma 8 The set $N$ is a normal subgroup of $G$. We have $\phi(N) \subset N$ and $\phi^{-1}(N)=$ $N$. Thus $\phi$ induces an endomorphism $[\phi / N](x N):=\phi(x) N$. The endomorphism $[\phi / N]: G / N \rightarrow G / N$ is injective, and we have $R(\phi)=R([\phi / N])$.

\section{PROOF}

(i) Let $x \in N, g \in G$. Then for some $n \in \mathbb{N}$ we have $\phi^{n}(x)=i d$. Therefore $\phi^{n}\left(g x g^{-1}\right)=\phi^{n}\left(g g^{-1}\right)=i d$. This shows that $g x g^{-1} \in N$ so $N$ is a normal subgroup of $G$.

(ii) Let $x \in N$ and choose $n$ such that $\phi^{n}(x)=i d$. Then $\phi^{n-1}(\phi(x))=i d$ so $\phi(x) \in N$. Therefore $\phi(N) \subset N$.

(iii) If $\phi(x) \in N$ then there is an $n$ such that $\phi^{n}(\phi(x))=i d$. Therefore $\phi^{n+1}(x)=i d$ so $x \in N$. This show that $\phi^{-1}(N) \subset N$. The converse inclusion follows from (ii).

(iv) We shal write $\mathcal{R}(\phi)$ for the set of $\phi$-conjugacy classes of elements of $G$. We shall now show that the map $x \rightarrow x N$ induces a bijection $\mathcal{R}(\phi) \rightarrow \mathcal{R}([\phi / N])$. Suppose $x, y \in G$ are $\phi$-conjugate. Then there is a $g \in G$ with $g x=y \phi(g)$. Projecting to the quotient group $G / N$ we have $\operatorname{gn} x N=y N \phi(g) N$, so $g N x N=y N[\phi / N](g N)$. This means that $x N$ and $y N$ are $[\phi / N]$-conjugate in $G / N$. Conversely suppose that $x N$ and $y N$ are $[\phi / N]$-conjugate in $G / N$. Then there is a $g N \in G / N$ such that $g N x N=y N[\phi / N](g N)$. In other words $\left.g x \phi(g)^{-1} y^{-1}\right)=i d$. Therefore $\phi^{n}(g) \phi^{n}(x)=$ $\phi^{n}(y) \phi^{n}(\phi(g))$.

This shows that $\phi^{n}(x)$ and $\phi^{n}(y)$ are $\phi$-conjugate. Howeever by lemma $1 x$ and $\phi^{n}(x)$ are $\phi$-conjugate as are $y$ and $\phi^{n}(y)$. Therefore $x$ and $y$ are $\phi$-conjugate .

(v) We have shown that $x$ and $y$ are $\phi$-conjugate iff $x N$ and $y N$ are $\phi / N$-conjugate. From this it follows that $x \rightarrow x N$ induces a bijection from $\mathcal{R}(\phi)$ to $\mathcal{R}([\phi / N])$. Therefore $R(\phi)=R([\phi / N])$.

Theorem 5 The Reidemeister number $R(\phi)$ is infinite if group $G / N$ is Gromov hyperbolic, non-elementary,torsion free, freely indecomposable and $\phi$ is any endomorphism of $G$ into itself.

Proof The proof follows from lemma 8 and theorems 3 and 4 . 


\section{Reidemeister number of continuous map}

Let now $X$ to be a connected, compact polyhedron and $f: X \rightarrow X$ to be a continuous map. Let a specific lifting $\tilde{f}: \tilde{X} \rightarrow \tilde{X}$ be chosen as reference. Then every lifting of $f$ can be written uniquely as $\gamma \circ \tilde{f}$, with $\gamma \in \Gamma$. So the elements of $\Gamma$ serve as coordinates of liftings with respect to the reference $\tilde{f}$. Now, for every $\gamma \in \Gamma$, the composition $\tilde{f} \circ \gamma$ is a lifting of $f$; so there is a unique $\gamma^{\prime} \in \Gamma$ such that $\gamma^{\prime} \circ \tilde{f}=\tilde{f} \circ \gamma$. This correspondence $\gamma \rightarrow \gamma^{\prime}$ is determined by the reference $\tilde{f}$, and is obviously a homomorphism.

Definition 1 The endomorphism $\tilde{f}_{*}: \Gamma \rightarrow \Gamma$ determined by the lifting $\tilde{f}$ of $f$ is defined by

$$
\tilde{f}_{*}(\gamma) \circ \tilde{f}=\tilde{f} \circ \gamma
$$

It is well known that $\Gamma \cong \pi_{1}(X)$. We shall identify $\pi=\pi_{1}\left(X, x_{0}\right)$ and $\Gamma$ in the following way. Pick base points $x_{0} \in X$ and $\tilde{x}_{0} \in p^{-1}\left(x_{0}\right) \subset \tilde{X}$ once and for all. Now points of $\tilde{X}$ are in 1-1 correspondence with homotopy classes of paths in $X$ which start at $x_{0}$ : for $\tilde{x} \in \tilde{X}$ take any path in $\tilde{X}$ from $\tilde{x}_{0}$ to $\tilde{x}$ and project it onto $X$; conversely, for a path $c$ starting at $x_{0}$, lift it to a path in $\tilde{X}$ which starts at $\tilde{x}_{0}$, and then take its endpoint. In this way, we identify a point of $\tilde{X}$ with a path class $\langle c\rangle$ in $X$ starting from $x_{0}$. Under this identification, $\tilde{x}_{0}=\langle e\rangle$ is the unit element in $\pi_{1}\left(X, x_{0}\right)$. The action of the loop class $\alpha=\langle a\rangle \in \pi_{1}\left(X, x_{0}\right)$ on $\tilde{X}$ is then given by

$$
\alpha=\langle a\rangle:\langle c\rangle \rightarrow \alpha \cdot c=\langle a \cdot c\rangle .
$$

Now we have the following relationship between $\tilde{f}_{*}: \pi \rightarrow \pi$ and

$$
f_{*}: \pi_{1}\left(X, x_{0}\right) \longrightarrow \pi_{1}\left(X, f\left(x_{0}\right)\right) .
$$

Lemma 9 Suppose $\tilde{f}\left(\tilde{x}_{0}\right)=\langle w\rangle$. Then the following diagram commutes:

$$
\begin{array}{ccc}
\pi_{1}\left(X, x_{0}\right) \underset{f_{*}}{\stackrel{f_{*}}{\longrightarrow}} & \pi_{1}\left(X, f\left(x_{0}\right)\right) \\
& \tilde{f}_{*} \searrow & \downarrow w_{*} \\
& \pi_{1}\left(X, x_{0}\right)
\end{array}
$$

where $w_{*}$ is the isomorphism induced by the path $w$.

In other words, for every $\alpha=\langle a\rangle \in \pi_{1}\left(X, x_{0}\right)$, we have

$$
\tilde{f}_{*}(\langle a\rangle)=\left\langle w(f \circ a) w^{-1}\right\rangle \text {. }
$$

Remark 1 In particular, if $x_{0} \in p(F i x(\tilde{f}))$ and $\tilde{x}_{0} \in F i x(\tilde{f})$, then $\tilde{f}_{*}=f_{*}$.

Lemma 10 Lifting classes of $f$ (and hence fixed point classes, empty or not) are in 1-1 correspondence with $\tilde{f}_{*}$-conjugacy classes in $\pi$, the lifting class $[\gamma \circ \tilde{f}]$ corresponding to the $\tilde{f}_{*}$-conjugacy class of $\gamma$. We therefore have $R(f)=R\left(\tilde{f}_{*}\right)$. 
We shall say that the fixed point class $p(F i x(\gamma \circ \tilde{f}))$, which is labeled with the lifting class $[\gamma \circ \tilde{f}]$, corresponds to the $\tilde{f}_{*}$-conjugacy class of $\gamma$. Thus $\tilde{f}_{*}$-conjugacy classes in $\pi$ serve as coordinates for fixed point classes of $f$, once a reference lifting $\tilde{f}$ is chosen.

Theorem 6 Reidemeister number $R(f)$ is infinite for any homeomorphism $f$ of a compact polyhedron $X$ with the non-elementary, Gromov hyperbolic fundamental group $\pi_{1}\left(X, x_{0}\right)$.

ProOF Proof immediately follows from theorem 3 and lemma 10 .

Theorem 7 The Reidemeister number $R(f)$ is infinite if fundamental group $\pi_{1}\left(X, x_{0}\right)$ is Gromov hyperbolic, non-elementary,torsion free, freely indecomposable and the map $f$ induces a monomorphism $\tilde{f}_{*}$ of $\pi_{1}\left(X, x_{0}\right)$ into itself.

Proof The proof follows from lemma 10 and theorem 4 .

Let now $f$ is any continious map of $X$. Let $N$ be the set of all nilpotent elements of $\pi_{1}\left(X, x_{0}\right)$ under $\tilde{f}_{*}$.

Theorem 8 For any map $f$ the Reidemeister number $R(f)$ is infinite if the group $\pi_{1}\left(X, x_{0}\right) / N$ is Gromov hyperbolic, non-elementary,torsion free, freely indecomposable.

Proof The proof follows from lemma 10 and theorem 5 .

\section{Pseudo-Anosov homeomorphism and asymptotic expansion}

Now we assume $X$ to be a compact surface of negative Euler characteristic and $f$ : $X \rightarrow X$ is a pseudo-Anosov homeomorphism, i.e. there is a number $\lambda>1$ and a pair of transverse measured foliations $\left(F^{s}, \mu^{s}\right)$ and $\left(F^{u}, \mu^{u}\right)$ such that $f\left(F^{s}, \mu^{s}\right)=\left(F^{s}, \frac{1}{\lambda} \mu^{s}\right)$ and $f\left(F^{u}, \mu^{u}\right)=\left(F^{u}, \lambda \mu^{u}\right)$. The mapping torus $T_{f}$ of $f: X \rightarrow X$ is the space obtained from $X \times[0,1]$ by identifying $(x, 1)$ with $(f(x), 0)$ for all $x \in X$. Take the base point $x_{0}$ of $X$ as the base point of $T_{f}$. According to van Kampen's Theorem, the fundamental group $\pi_{1}\left(T_{f}, x_{0}\right)$ is obtained from $\pi_{1}\left(X, x_{0}\right)$ by adding a new generator $z$ and adding the relations $z^{-1} g z=\tilde{f}_{*}(g)$ for all $g \in \pi_{1}\left(X, x_{0}\right)$, where $z$ is the generator of $\pi_{1}\left(S^{1}, x_{0}\right)$. This means that $\pi_{1}\left(T_{f}, x_{0}\right)$ is a semi-direct product of $\pi_{1}\left(X, x_{0}\right)$ with $Z$.

From Lemma 2 it follows that two elements $x, y$ of $\pi_{1}\left(X, x_{0}\right)$ are $\tilde{f}_{*}$-conjugate if and only if $x z$ and $y z$ are conjugate in the usual sense in $\pi_{1}\left(T_{f}, x_{0}\right)$. Therefore $R(f)=R\left(\tilde{f}_{*}\right)$ is the number of usual conjugacy classes in the coset $\pi_{1}\left(X, x_{0}\right) \cdot z$ of $\pi_{1}\left(X, x_{0}\right)$ in $\pi_{1}\left(T_{f}, x_{0}\right)$. There is a canonical projection $\tau: T_{f} \rightarrow R / Z$ given by $(x, s) \mapsto s$. This induces a map $\pi_{1}(\tau): G=\pi_{1}\left(T_{f}, x_{0}\right) \rightarrow Z$.

We see that the Reidemeister number $R(f)$ is equal to the number of homotopy classes of closed paths $\gamma$ in $T_{f}$ whose projections onto $R / Z$ are homotopic to the path

$$
\begin{aligned}
\sigma:[0,1] & \rightarrow R / Z \\
s & \mapsto s .
\end{aligned}
$$


Corresponding to this, there is a group-theoretical interpretation of $R(f)$ as the number of usual conjugacy classes of elements $\gamma \in \pi_{1}\left(T_{f}\right)$ satisfying $\pi_{1}(\tau)(\gamma)=z$.

Lemma 11 [15, 10] The interior of the mapping torus Int $\left(T_{f}\right)$ admits a hyperbolic structure of finite volume if and only if $f$ is isotopic to a pseudo-Anosov homeomorphism.

So, if the surface $X$ is closed and $f$ is isotopic to a pseudo-Anosov homeomorphism, the mapping torus $T_{f}$ can be realised as a hyperbolic 3 -manifold, $H^{3} / \pi_{1}\left(T_{f}, x_{0}\right)$, where $H^{3}$ is the Poincare upper half space $\left\{(x, y, z): z>0,(x, y) \in R^{2}\right\}$ with the metric $d s^{2}=\left(d x^{2}+d y^{2}+d z^{2}\right) / z^{2}$.

The closed geodesics on a hyperbolic manifold are in one-to-one correspondence with the free homotopy classes of loops. These classes of loops are in one-to-one correspondence with the conjugacy classes of loxodromic elements in the fundamental group of the hyperbolic manifold.

This correspondence allows Ch. Epstein ( see [4, p.127) to study the asymptotics of such functions as $p_{n}(x)=\#\{$ primitive closed geodesics of length less than $x$ represented by an element of the form $\left.g z^{n}\right\}$ using the Selberg trace formula. A primitive closed geodesic is one which is not an iterate of another closed geodesic.

Phillips and Sarnak [12] have generalised results of Epstein in the following way. Let $M$ be a compact Riemannian manifold of negative curvature. Let $\psi: G \rightarrow \Lambda$ be a surjective homomorphism of the fundamental group $G$ of $M$ onto an abelian group $\Lambda$. Let $r$ be the rank of $\Lambda$ and let $m$ be the order of the torsion of $\Lambda$; i.e., $\Lambda$ is isomorphic to $Z^{r} \cdot F, F$ being finite of order $m$. For $\beta \in \Lambda$ let $p_{\beta}(x)$ be the number of primitive closed geodesics $\gamma$ on $M$ of length at most $x$ for which $\psi(\gamma)=\beta$.

Theorem 9 12 We have the asymptotic expansion

$$
p_{\beta}(x) \sim \frac{e^{(n-1) x}}{m x^{r / 2+1}}\left(C_{0}+\frac{C_{1}}{x}+\frac{C_{2}}{x^{2}}+\ldots\right)
$$

as $x \rightarrow \infty$. Here $C_{0}$ is independent of $\beta ; C_{0}$ is the determinant of a certain period matrix of harmonic 1-forms on $M$.

The proof of this result makes routine use of the Selberg trace formula. If $\Lambda=$ $H_{1}(M, Z)$ then theorem 9 gives the asymptotic of the number of primitive closed geodesics of length at most $x$ lying in fixed homology class. In the more general case of variable negative curvature similar asymptotic was obtained by Pollicott and Sharp [13]. They used dynamical approach based on the geodesic flow. We will only need an asymptotic for $p_{1}(x)$. Note, that closed geodesics which are represented by an element of the form $g z$ are automatically primitive, because they wrap exactly once around the mapping torus (once around generator $z$ ). Taking $\Lambda=Z$, a surjective homomorphism $\pi_{1}(\tau): G=\pi_{1}\left(T_{f}, x_{0}\right) \rightarrow Z$ and $\beta=z$-generator of group $Z$ in theorem 9 we have the following asymptotic expansion [1, 12, 13, 国]

$$
p_{1}(x)=\frac{e^{h x}}{x^{3 / 2}}\left(\sum_{n=0}^{N} \frac{C_{n}}{x^{n / 2}}+o\left(\frac{1}{x^{N / 2}}\right)\right),
$$


for any $N>0$, where $h=\operatorname{dim} T_{f}-1=2$ is the topological entropy of the geodesic flow on the unit-tangent bundle $S T_{f}$, the constant $C_{0}>0$, constants $C_{n}$ vanish if $n$ is odd. So in particular, using $N=1$ we have

$$
p_{1}(x) \sim C_{0} \frac{e^{h x}}{x^{3 / 2}}, x \rightarrow \infty
$$

Since $C_{0}>0$ we see that $p_{1}(x)$ goes to infinity as $x$ does.

Now, using one-to-one correspondences in lemma 2 and lemma 10 we define the norm of a fixed point class, or of the corresponding lifting class, or of the corresponding twisted conjugacy class $\{g\}_{\tilde{f}_{*}}$ in the fundamental group of the surface $\pi_{1}\left(X, x_{0}\right)$, as the length of the primitive closed geodesic $\gamma$ on $T_{f}$, which is represented by an element of the form $g z$. So, for example, the norm function $l^{*}$ on the set of twisted conjugacy classes equals $l^{*}=l \circ B$, where $l$ is length function on geodesics $(l(\gamma)$ is the length of the primitive closed geodesic $\gamma$ ) and $B$ is a bijection between the set of twisted conjugacy classes $\{g\}_{\tilde{f}_{*}}$ in the fundamental group of the surface $\pi=\pi_{1}\left(X, x_{0}\right)$ and the set of closed geodesics represented by an elements of the form $g z$ in the fundamental group $G:=\pi_{1}\left(T_{f}, x_{0}\right)$. We introduce the following counting functions $\operatorname{FPC}(x)=\#\{$ fixed point classes of $f$ of norm less than $x\}$, $\mathrm{L}(x)=\#\{$ lifting classes of $f$ of norm less than $x\}$, $\mathrm{Tw}(x)=\#$ twisted conjugacy classes for $\tilde{f}_{*}$ in the fundamental group of surface of norm less than $x\}$. We have following asymptotic expansion

Theorem 10 Let $X$ be a closed surface of negative Euler characteristic and let $f$ : $X \rightarrow X$ be a pseudo-Anosov homeomorphism. Then

$$
F P C(x)=L(x)=T w(x)=\frac{e^{2 x}}{x^{3 / 2}}\left(\sum_{n=0}^{N} \frac{C_{n}}{x^{n / 2}}+o\left(\frac{1}{x^{N / 2}}\right)\right)
$$

where the constant $C_{0}>0$ depends on the volume of the hyperbolic 3-manifold $T_{f}$, and the constants $C_{n}$ vanish if $n$ is odd.

Proof The proof follows from lemmas 2 and 10 and asymptotic expansion (1).

Corollary 1 For pseudo-Anosov homeomorphisms of closed surfaces the Reidemeister number is infinite.

As we see, Theorem 10 gives analytical proof of Lemma 3 in the case of pseudo-Anosov automorphism of surface group.

Theorem 10 indicates also, that we can develop an analog of Selberg theory for twisted conjugacy classes of group automorphism. We have the following question in this direction

Question 1 Is there an asymptotic of counting function for twisted conjugacy classes of hyperbolic automorphism of Gromov hyperbolic group? 


\section{References}

[1] N. Anantharaman, Precise counting results for closed orbits of Anosov flows. Preprint, 1998.

[2] M. Bestvina, M. Feighn, A combination theorem for negatively curved groups, Jour. Diff. Geom. 35(1992), 85-101.

[3] M. Cohen, M. Lustig, On the dynamics and the fixed subgroup of a free group automorphism. Inventiones Mathematicae, 196(1989), 613-638.

[4] C. Epstein, The spectral theory of geometrically periodic hyperbolic 3-manifolds. Memoirs of the AMS, vol. 58, number 335, 1985.

[5] Fel'shtyn A.L. ,Hill R.The Reidemeister zeta function with applications to Nielsen theory and a connection with Reidemeister torsion. K-theory, v.8, n.4, 1994, 367393.

[6] Fel'shtyn A.L. Dynamical zeta functions, Nielsen theory and Reidemeister torsion. Memoirs of the American Mathematical Society, v.147, no.699, September 2000, 146 pages.

[7] Fel'shtyn A.L. Zeta functions, 3-manifolds and asymptotics in Nielsen theory. Topology and its Applications, to appear.

[8] G. Levitt, M. Lustig, Most automorphisms of a hyperbolic group have very simple dynamics. Ann. Sc. ENS 33(2000), 507-517.

[9] M. Gromov, Hyperbolic groups, in Gersten S. M. (Ed), Essays in Group theory, MSRI public., vol. 8, Springer, 1987, p. 75-263.

[10] J.-P. Otal, Le theoreme d'hyperbolisation pour les varietes fibrees de dimension 3. Asterisque vol. 235, 1996.

[11] F. Paulin, Sur les automorphismes exterieurs des groupes hyperboliques. Ann. Sc. ENS 30(1997), 147-167.

[12] R. Phillips, P. Sarnak, Geodesics in homology classes. Duke math. journal, v.55, n.2, 1987, p. 287-297.

[13] M. Pollicott, R. Sharp, Asymptotic expansions for closed orbits in homology classes. Preprint ESI 594, 1998, Vienna.

[14] Z. Sela, Structure and rigidity in (Gromov) hyperbolic groups and discrete groups in rank 1 Lie groups. II. GAFA, vol. 7(1997), 561-593.

[15] W. Thurston, Hyperbolic structures on 3-manifolds, II: surface groups and 3manifolds which fibers over the circle. Preprint. 
Institut für Mathematik,

Ernst-Moritz-Arndt-Universität Greifswald

Jahn-strasse 15a, D-17489 Greifswald, Germany.

E-mail address: felshtyn@mail.uni-greifswald.de 